\title{
Analysis on Ability of Spaceborne Synthetic Aperture Radar Detecting Cavern in Bare Hillslope
}

\author{
C.-C. SHI ${ }^{1,2}$, X.-J. Zhang ${ }^{1}$, Zheng Liu ${ }^{2}$, Y.-H. Sun ${ }^{2}$ \\ ${ }^{1)}$ Erqi Road 145, Wuhan, Hubei, China (shicuncheng @126.com) \\ ${ }^{2)}$ State Key Laboratory for Disaster Prevention \& Mitigation of Explosion \& Impact, PLA University of Science \& Technology, \\ Nanjing, Jiangsu, China (zanxiaojuan@126.com)
}

\begin{abstract}
Spaceborne Synthetic Aperture Radar has certain capacity of detecting underground things. According to the facet theory and radar image simulation method, the mountain slope with the underground cavern has been divided into many grids based on the radar resolution criterion. The radar echo intensity calculation formula has been got by considering the electromagnetic propagation in the rock mass and on the cavern roof. According to the calculation formula, the radar echo intensity calculating program has been done by the matlab sofrware, then the maximum detecting depth of the SAR has been studied with the criterion of minimum detectable signal power. Numerical examples show that the minimum rock thickness of the underground cavern to satisfy the anti-detecting demand is $2100 \mathrm{~mm}$ by taking into account the current SAR detectability and rock electric property constant can influence the maximum detecting depth of the SAR very much by depth increasing together with the electric property constant raises.
\end{abstract}

Key words — Synthetic Aperture Radar, underground cavern, radar echo intensity, minimum detectable signal power

\section{星载合成孔径雷达对裸露岩坡洞室探测能力分析}

\author{
施存程 ${ }^{1,2} \quad$ 张小娟 $^{1} \quad$ 刘峥 $^{2} \quad$ 孙云厚 $^{2}$ \\ 1) 二七路 145 号，武汉，湖北，中国; \\ 2) 解放军理工大学爆炸冲击防灾减灾国家重点实验室, 南京, 江苏, 中国
}

摘 要 星载合成孔径雷达具备一定的探地能力, 根据 “小平面理论”, 按照雷达图像模拟方法将洞室所在的山坡坡面以雷达分辨 率为标准划分网格, 考虑电磁波在岩石、洞室顶板的传播, 得到了雷达回波强度计算公式, 并编写回波强度计算程序, 以最小可检测 信号功率为标准研究了合成孔径雷达最大探测深度。算例表明, 考虑当前合成孔径雷达探测能力, 地下洞室岩石覆盖层最小厚度取 $2100 \mathrm{~mm}$ 可以满足抗探测要求; 岩石电性参数对合成孔径雷达最大探测深度影响较大, 随着介电常数的增加, 探测深度显著增加。

关键词＼cjkstart合成孔径雷达，地下洞室，回波强度，最小可检测信号功率

1. 引言

星载合成孔径雷达具备一定的探地能力, 人们通过 分析 SIR-A 图像发现了撒哈拉大沙漠东部沙层覆盖下的 古河道和古人类遗迹 ${ }^{[1]}$, 邵芸发现 SIR-C/X-SAR 可以探 测到阿拉善地区深度大于 $2.82 m$ 的地物 ${ }^{[2]}$ 。为获得高分 辨率图像, 合成孔径雷达对重点地区一般采用聚束式工 作模式, 能获得分辨率 $0.3 \sim 1 m$ 的块状雷达图像 ${ }^{[3]}$ 。目 前, 星载合成孔径雷达主要采用 $\mathrm{X} 、 \mathrm{~L}$ 波段, 有多种极 化方式, 对地遥感能力很强。为提高探测地下洞室能力,

国家自然科学基金项目支持 (资助号: 51309233)
有关机构还重点研究了超宽带合成孔径雷达 ${ }^{[4,5]}$, 并组织 了多次实验, 发现其对粘土区穿透深度为 $2 \sim 3 m$, 对干 燥沙漠区可达几十米。目前, 最具代表意义的是 CARABAS (20 MHz $\sim 90 M H z)$, 据报道可探测到地下 5 $10 m$ 的目标。因此, 有必要对合成孔径雷达探测地下洞 室能力进行分析。

2. 分析思路

根据合成孔径雷达探测原理绘制探测地下洞室示意

图, 如图 1。电磁波在坡面上发生反射和透射, 反射波 
返回至雷达天线; 透射波 $O_{01} P$ 在岩体中传播, 当遇到洞 室顶板时产生反射, 反射波 $P O_{02}$ 经坡面透射后返回雷达 天线, 返回至雷达天线的电磁波有两种: 坡面直接返回 的电磁波、经洞室顶板二次反射后返回的电磁波, 前者 相当于不设洞室坡面产生的回波, 后者相当于目标回波。

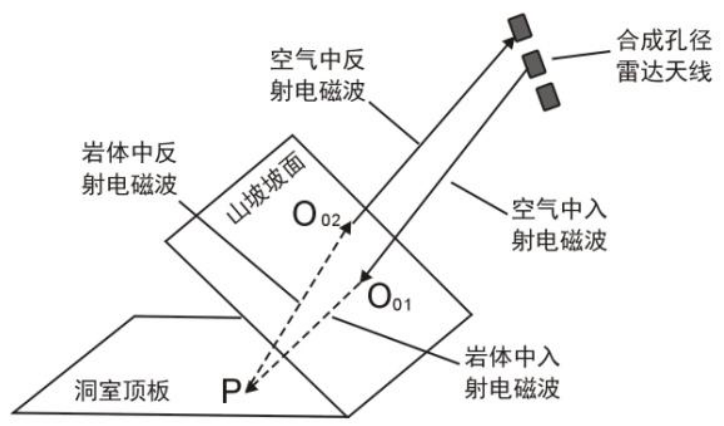

图 1 合成孔径雷达探测地下洞室示意图

当在合成孔径时间内目标回波功率大于最小可检测 信号功率时, 回波功率不会淹没在噪声中, 雷达才会探 测到回波。因此, 该问题分析思路如图 2。

合成孔径雷达的最小可检测功率为:

$$
P_{r \text { min }}=(S / N)_{\min } k T_{s} B_{n}
$$

式中: $(S / N)_{\min }$ 为最小可检测信噪比, 倍; $k$ 为玻耳兹 曼常数; $B_{n}$ 为接收机检波前的噪声带宽, $\mathrm{Hz} ; T_{s}$ 为接 收系统噪声温度, $k$ 。

当合成孔径时间内洞顶回波总功率小于式 (2) 计算 的功率值时, 雷达不会探测到回波差值。

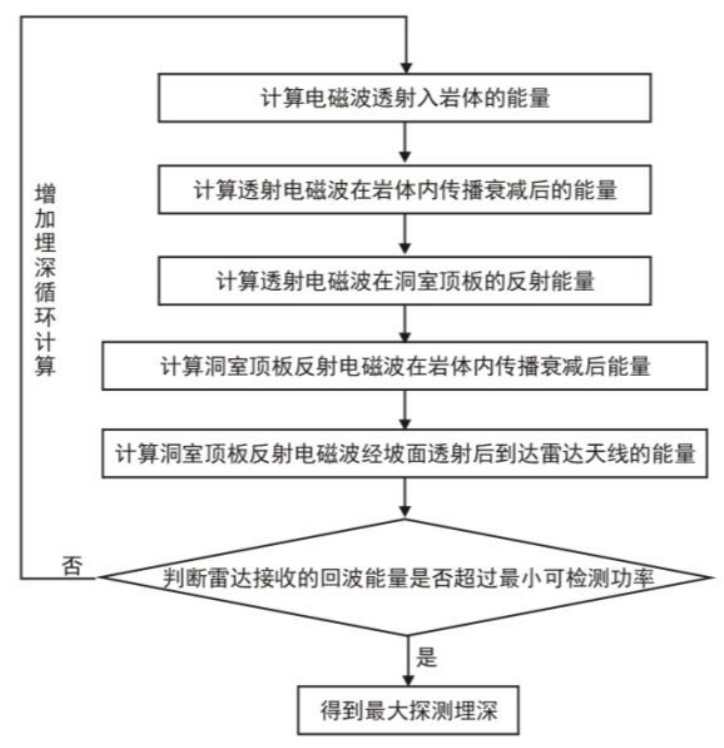

图 2 合成孔径雷达探测地下洞室分析思路

$$
W_{r b} \cdot T_{a} \cdot P R F<(S / N)_{\min } k T_{s} B
$$

式中: $W_{r b}$ 为雷达接收到的洞顶处山坡回波功率, $w ; T_{a}$ 为合成孔径时间, $s ; P R F$ 为脉冲重复频率, $H z$ 。

式 (2) 即为判断合成孔径雷达最大探测埋深的准 则, 因此问题的关键在于求洞顶处山坡的回波强度。

\section{3. 回波强度计算}

\section{1 基本假设}

各散射单元的回波相位互不相干，可将回波功率相 加取均值计算平均回波强度 ${ }^{[6]}$ 。回波是表面散射和内部 体散射的合成, 这使雷达散射十分复杂。因此, 假设:

1）坡面为粗粮平面，洞室内表面为光滑面;

2）岩石为均匀介质，电性参数恒定。

\section{2 回波强度计算步骤}

根据 “小平面理论” (FACET THEORY) ${ }^{[7]}$ 及雷达 图像模拟思路, 以分辨单元尺寸为标准将地面划分成网 格, 其中每一格就是雷达图像上一个像素对应的地面单 元, 通过计算每个地面单元的散射回波强度就可转换成 图像灰度值 ${ }^{[8]}$ 。如图 3, 将地下洞室外山坡划分成分辨 单元尺寸的网格，洞顶网格划分按山坡网格的投影取。 电磁波经山坡表面透射进入岩石后，在洞室分界面发生 镜面反射后返回山坡表面, 对每个网格的回波强度取平 均后，按雷达方程计算雷达天线处的回波功率:

1)计算洞室某网格的电磁波入射与反射路径;

2)计算在洞室顶部某网格发生反射时的电磁波能量 和反射回山坡上某网格的能量;

3)对山坡上某网格按上述思路计算洞顶所有网格反 射回的能量, 再计算回波强度。

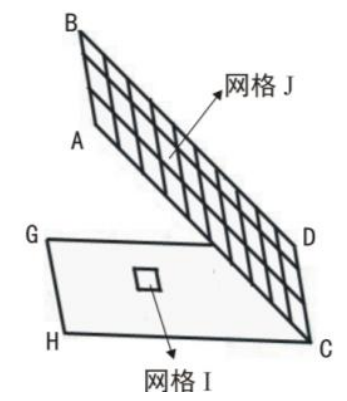

图 3 回波强度计算网格划分

3.3 岩体内电磁波传播路径

岩体内电磁波经历入射和反射两个过程，如图 4, 


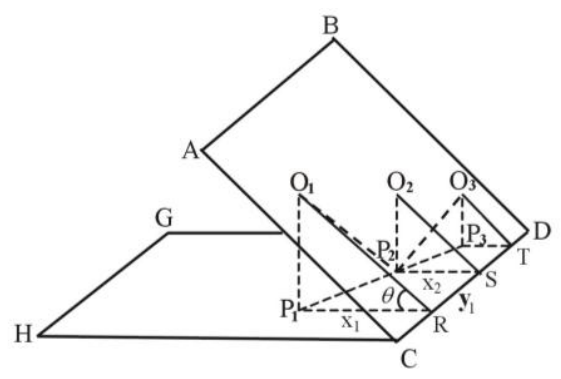

图 4 电磁波入射与反射路径

$O_{1} 、 O_{2} 、 O_{3}$ 分别为山坡上某三个网格的中心, $P_{1} 、 P_{2}$ 、 $\mathrm{P}_{3}$ 为洞室顶部由对应于 $\mathrm{O}_{1} 、 \mathrm{O}_{2} 、 \mathrm{O}_{3}$ 三网格投影的中心, $x_{1} 、 x_{2}$ 分别是 $P_{1} 、 P_{2}$ 到 $R S T$ 的距离。由斯涅耳定律, 得:

入射电磁波路径:

$$
R_{1}=\mathrm{O}_{3} \mathrm{P}_{2}=\frac{x_{2}}{2 x_{1}-x_{2}} \sqrt{x_{1}^{2} \tan ^{2} \theta+y_{1}^{2}+\left(x_{2}-x_{1}\right)^{2}}
$$

反射电磁波路径:

$$
R_{2}=\mathrm{P}_{2} \mathrm{O}_{1}=\sqrt{x_{1}^{2} \tan ^{2} \theta+y_{1}^{2}+\left(x_{2}-x_{1}\right)^{2}}
$$

由式 (3) 知, 只有满足 $2 x_{1}-x_{2}>0$ 条件的洞顶网格 才能将电磁波反射到已知山坡网格上。因此, 以 $2 x_{1}-x_{2}>0$ 为条件计算反射电磁波能量, 再以最小可检 测信号功率确定最大探测深度。

\section{4 雷达接收的反射能量}

根据电磁波传播理论, 透射功率为 $W(0)$ 的电磁波在 传播长度 $R_{l}$ 处的功率 ${ }^{\left[{ }^{[}\right]}$为:

$$
W\left(R_{l}\right)=W(0) e^{-2 \alpha R_{l}}
$$

当两种介质的相对介电常数分别为 $\varepsilon_{1}, \varepsilon_{2}$, 电磁波 在二者界面反射时, 其功率反射系数的估算式 ${ }^{[9]}$ 为:

$$
P_{r}=\left(\frac{\sqrt{\varepsilon_{1}}-\sqrt{\varepsilon_{2}}}{\sqrt{\varepsilon_{1}}+\sqrt{\varepsilon_{2}}}\right)^{2}
$$

由雷达方程, 山坡单个网格透射的电磁波功率为:

$$
W_{t s}=\frac{W_{t} G_{t}}{4 \pi R^{2}} A_{\text {small }}\left(1-P_{r}\right)
$$

式中: $A_{\text {small }}$ 为山坡网格面积, $\mathrm{m}^{2} ; R$ 为山坡到星载雷达 天线的距离, $m$ 。

距离山坡网格 $R_{I}$ 处的洞顶网格的电磁波功率为:

$$
W_{d m}=W_{t s} \cdot \frac{A_{d m}}{2 \pi R_{1}^{2}} e^{-2 \alpha R_{1}}
$$

式中: $A_{d m}$ 为洞顶某网格面积, $m^{2} ; \alpha$ 为衰减系数, $N P / m$ 。 电磁波在洞顶某网格处反射到 $R_{2}$ 处山坡某网格的 功率为:

$$
W_{f a n-i}=P_{r} \cdot W_{t s} \cdot \frac{A_{d m}}{2 \pi R_{1}^{2}} e^{-2 \alpha R_{1}} e^{-2 \alpha R_{2}}
$$

山坡上某网格接收的能量由洞顶所有网格回波能 量叠加而得, 即:

$$
W_{\text {shan }-j}=\sum_{i=1}^{m} W_{f a n-i}
$$

则经洞顶反射后山坡的平均回波功率为:

$$
W_{\text {shan }}=\frac{\sum_{j=1}^{n} W_{\text {shan }-j}}{n}
$$

雷达天线处接收的洞顶反射回的能量为:

$$
W_{r b}=\left(1-P_{r}\right) \cdot W_{\text {shan }} \cdot G_{t} \lambda^{2} /(4 \pi R)^{2}
$$

\section{4. 算例与讨论}

\section{1 计算条件}

结合已知合成孔径雷达有关资料 ${ }^{[3,10]}$ 选取计算参 数, 见表 1 。常见介质电磁波传播参数 ${ }^{[9,11]}$ 见表 2 , 地下 洞室为截面 $4 m \times 4 m$ 的箱型洞室, 山坡坡角 $60^{\circ}$ 。

\section{2 计算结果分析}

根据式 (2) 和回波功率计算程序, 得出满足不同 最小检测信噪比要求的最大探测深度, 见表 3 。

根据文献[7], 雷达在 0.9 检测概率情况下最小可检 测信噪比为 $-13 d B$ 。Radarsat 合成孔径雷达的最小可检测 信噪比大于 $-10 d B$ 。因此, 以 $-20 d B$ 为最小可检测信噪比 标准计算岩层覆盖厚度可以满足地下洞室抗合成孔径雷 达探测要求, 此时合成孔径雷达对以上 8 类岩石的最大 探测深度不超过 $2.1 \mathrm{~m}$ 。

\section{3 影响因素分析}

从表 3 可知, 岩石电性参数是影响厚度的主要因素。 同种岩石因电性参数造成的厚度差异达 10 倍以上, 电导 率比相对介电常数对结果影响更明显。干性介质由于电 导率较小, 电磁波能量衰减少, 回波强度大, 介质由于 含水量增加使电导率增大, 电磁波能量衰减幅度大, 回 波强度较小, 使厚度明显降低, 最大仅为 $700 \mathrm{~mm}$, 对湿 灰岩、湿沙土、饱和页岩而言厚度则不超过 $50 \mathrm{~mm}$ 。 
表 1 合成孔径雷达参数

\begin{tabular}{|c|c|c|c|c|c|c|c|c|c|c|}
\hline $\begin{array}{c}\text { 飞行高度 } \\
/ \mathrm{km}\end{array}$ & $\begin{array}{c}\text { 天线增益 } \\
/ \mathrm{dB}\end{array}$ & $\begin{array}{c}\text { 峰值功率 } \\
/ \mathrm{w}\end{array}$ & $\begin{array}{c}\text { 雷达波长 } \\
/ \mathrm{m}\end{array}$ & $\begin{array}{c}\text { 雷达波频率 } \\
/ \mathrm{GHz}\end{array}$ & $\begin{array}{c}\text { 分辨率 } \\
/ \mathrm{m}^{2}\end{array}$ & $\begin{array}{c}B_{n} \\
/ \mathrm{MHz}\end{array}$ & $\begin{array}{c}P R F \\
/ H z\end{array}$ & $\begin{array}{c}\text { 合成孔径 } \\
\text { 时间 } / \mathrm{s}\end{array}$ & $\begin{array}{c}\text { 系统噪声 } \\
/ k\end{array}$ & $\begin{array}{c}(S / N)_{\min } \\
/ d B\end{array}$ \\
\hline 670 & 36.4 & 4400 & 0.24 & 1.25 & 1 & 10 & 2000 & 3.02 & 580 & $-10 \sim-30$ \\
\hline
\end{tabular}

表 2 常见岩石介质的相对介电常数

\begin{tabular}{|c|c|c|c|c|c|c|c|c|c|c|}
\hline \multirow{2}{*}{ 岩石类别 } & \multicolumn{2}{|c|}{ 花岗岩 } & \multicolumn{2}{|c|}{ 灰 岩 } & \multicolumn{2}{c|}{ 砂 岩 } & \multicolumn{2}{c|}{ 沙 } & \multicolumn{2}{|c|}{ 页岩 } \\
\cline { 2 - 12 } & 干 & 湿 & 干 & 湿 & 干 & 湿 & 干 & 湿 & 干 & 饱和 \\
\hline 相对介电常数 & 4 & 7 & 7 & 8 & 3.5 & 7.5 & 4 & 15 & 5 & 12 \\
\hline 电导率 $(\mathrm{S} / \mathrm{m})$ & $10^{-5}$ & 0.005 & $1.625 \times 10^{-3}$ & 0.05 & $5.5 \times 10^{-6}$ & $10^{-2}$ & $10^{-4}$ & 0.05 & $10^{-3}$ & 0.05 \\
\hline
\end{tabular}

表 3 合成孔径雷达对不同岩石的最大探测深度 $(\mathrm{mm})$

\begin{tabular}{|c|c|c|c|c|c|c|c|c|c|c|}
\hline $\begin{array}{c}\text { 最小可检测 } \\
\text { 信噪比/dB }\end{array}$ & 干花岗岩 & 湿花岗岩 & 干灰岩 & 湿灰岩 & 干砂岩 & 湿砂岩 & 干沙 & 湿沙 & 干页岩 & 饱和页岩 \\
\hline-5 & 100 & 50 & 50 & 50 & 150 & 50 & 100 & 50 & 50 & 50 \\
\hline-10 & 250 & 50 & 100 & 50 & 400 & 50 & 250 & 50 & 100 & 50 \\
\hline-15 & 850 & 50 & 250 & 50 & 950 & 50 & 700 & 50 & 250 & 50 \\
\hline-20 & 1900 & 150 & 550 & 50 & 2100 & 50 & 1550 & 50 & 600 & 50 \\
\hline-25 & 3600 & 300 & 1050 & 50 & 3950 & 150 & 3000 & 50 & 1200 & 50 \\
\hline-30 & 6100 & 600 & 1800 & 50 & 6600 & 300 & 5200 & 50 & 2300 & 50 \\
\hline
\end{tabular}

\section{5. 结论}

1)覆盖层厚度不小于 $2100 \mathrm{~mm}$ 的裸露岩坡内部洞室 可以满足抗星载合成孔径雷达探测要求。

2) 岩石电性参数对探测深度影响较大, 随着介电 常数增加, 合成孔径雷达的探测深度显著增加。

\section{参考文献(References)}

[1] J. F. McCauley, G. G. Schabe, C. S. Breed, et a, "Subsurface valleys and geoarchaeology of the eastern Sahara revealed by Shuttle Radar," Science, vol. 218, pp. 1004-1020, 1982.

[2] Yun Shao, Space shuttle imaging radar SIR-C/X-SAR penetration experimental analysis. Institute of Remote Sensing Applications Chinese Academy of Sciences. In: Remote Sensing Science Progress, Beijing: Science Publishing House, 1995.

[3] X.-K. Yuan, Introduce to the Spaceborne Synthetic Aperture Radar, Beijing: National Defence Industry Publishing House, 2003.

[4] Z.-M. Zhou,X.-T. Huang, "Penetration performance analysis of VHF/UHF ultra-wideband synthetic aperture radar," Systems Engineering and Electronics, vol. 25, no.11, pp. 1336-1339,
November 2003.

[5] X.-T. Huang, D.-N. Liang, Z.-M. Zhou, "Frequency analysis and selection of penetrating foliage UWB SAR," Modern Radar, vol. 24, no. 3, pp. 5-8, May 2002.

[6] L.-P. Zhao, "The forth imaging radar microwave remote sensing knowledge introducing: target scattering properties," Remote Sensing for Land \& Resources, no. 12, pp. 60-63, December 1991.

[7] M.-I. Skolnik, Radar Handbook, Second ed, The McGraw-Hill Companies, 1990.

[8] N. Shu, Principles of Microwave Remote Sensing, Wuhan: Wuhan University publishing house, 2003.

[9] H.-F. Nie, "The application of airborne SLA image in surveying fossil river course," Remote Sensing for Land \& Resources, no. 51, pp. 15-18, March 2002.

[10] S.-F. Zeng, S.-X. Liu, Z.-J. Wang, et al., The method of ground-penetrating radar theory and application, Beijing: Science Publishing House, 2006.

[11] J.-X. Huang, D.-T. Ding, W.-M. Lei, "Characteristics of azimuth resolution of distributed space-borne SAR system," Modern Radar, vol. 26, no. 9, pp. 43-45, September 2004. 